Cite this article: Pacurar M, Jurca AM, Roman D, Bud E, Zetu I, Vata I. Nonextraction methods for creating space in orthodontic therapy. Stoma Edu J. 2014; 1(1):18-21.

\section{NONEXTRACTION METHODS FOR CREATING SPACE IN ORTHODONTIC THERAPY}

https://doi.org/10.25241/stomaeduj.2014.1(1).art.3

\section{Mariana Păcurar ${ }^{1 a}$, Ana Maria Jurcă'c, Doru Roman ${ }^{1 \mathrm{~b} *}$, Eugen Bud'c, Irina Nicoleta Zetu ${ }^{2 b}$, Ioana Vâțăcc}

1. Orthodontic Department, Faculty of Dentistry, University of Medicine and Pharmacy of Târgu-Mureş, Târgu-Mureș, Romania

2. Orthodontic Department, Faculty of Dentistry "Gr.T.Popa" University of Medicine and Pharmacy, Jassy, Romania

a. DDS, PhD, Professor, Dean of Faculty of Dentistry b. DDS, PhD, Lecturer c. DDS, Assistant Professor
Received: 19 November 2013 Accepted: 11 March 2014

\section{* Corresponding author:}

Lecturer Doru Roman, DDS, PhD Orthodontic Department, Faculty of Dentistry, University of Medicine and Pharmacy of TârguMureş, Târgu-Mures, Romania. 38 Gh. Marinescu Str., RO-540139, Târgu-Mureş, Romania. Tel/Fax: +40265210407. e-mail: tudorroman2000@yahoo.com

\section{Abstract}

Introduction: Molar distalization is an alternative treatment method in dento-maxillary anomalies, to avoid extraction especially in low angle cases. The orthodontic literature indicates that upper molar distalization is a tipping movement, combined with mesiobuccal rotation and buccally-crown torque. The aim of the study was to analyze the advantages to create space during upper first molar distalization movement, by using different devices. We used this method in skeletal Angle Class II, dental Class II/2 malocclusion with crowding and low profile.

Methodology: The study consisted of a retrospective statistical analysis on 435 patients aged 11-13 years treated with fixed appliances (straight wire technique), between 20092012. The patients were divided in two groups: group $A(83)$ who worn distalization devices and group B (352) who did not. Group B was divided in: B1 (278) with other nonextraction appliances and B2 (74) with extraction during orthodontic treatment. Results: Upper molar distalization was successful in $45 \%$ of the cases, the values of the space being: 2,13-2,33 mm, by tipping movement. Bodily distal upper molar movement was successfully obtained only when the rotational axis is at infinite and the compressive stress is homogeneously distributed in the periodontal ligament. The success rate depended on: eruption of the second molar, overjet and overbite size.

\section{Conclusions:}

1. Molar distalization is a challenge in orthodontic treatment and is indicated for Angle Class II, crowding and low angle (extraction makes the profile worse).

2. Molar distalization depends on the position of the second molar and this technique is not singular, but associated with multibracket appliance .

Key words: distalization, second molar, class II, extraction, fixed appliances.

\section{Introduction}

Modern orthodontic therapy attempts, whenever possible, a nonextraction treatment, with convenient means for the patient, which would allow current activities and it would not affect facial harmony (1).

In this context, molar distalization is an useful treatment method in obtaining arcade space, especially in anomalies Angle Class II/2 with accentuated retrognatic profile and hipodivergent growth pattern, cases where extraction would obviously create aesthetic facial damage (2).

The authors propose in this paper an assessment of the molar distalization method in comparison with other nonextraction therapy methods (expansion, frontal protrusion and stripping).

\section{Methods}

We conducted a retrospective statistical study on a sample of 435 patients, aged between 11-13 years, who were treated at the Orthodontic Department of the Faculty of Dentistry in Târgu Mureș in the period 2009-2012, for various malocclusions.

The initial sample was divided into two subgroups: group A - 83 patients average age 11,25 with upper or lower molar distalization. The following parameters were evaluated:

- duration of treatment;

- type of distalization:

- type of used appliance;

- obtained results. 


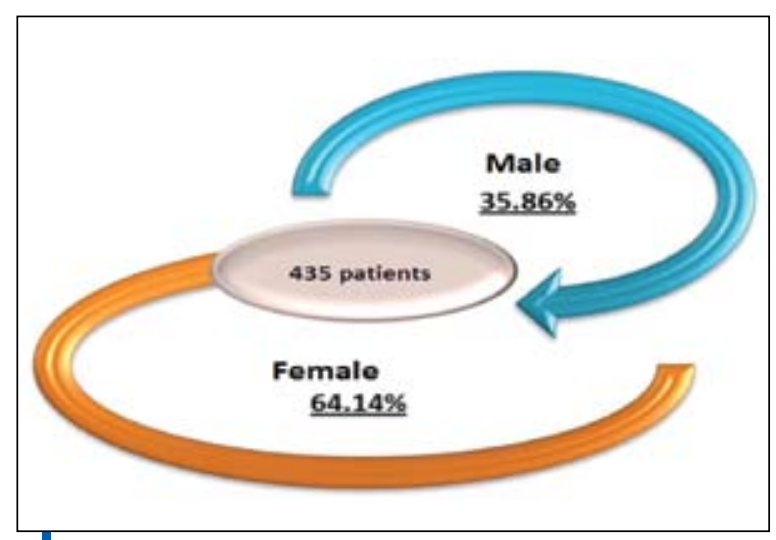

Figure 1. Cases distributrion

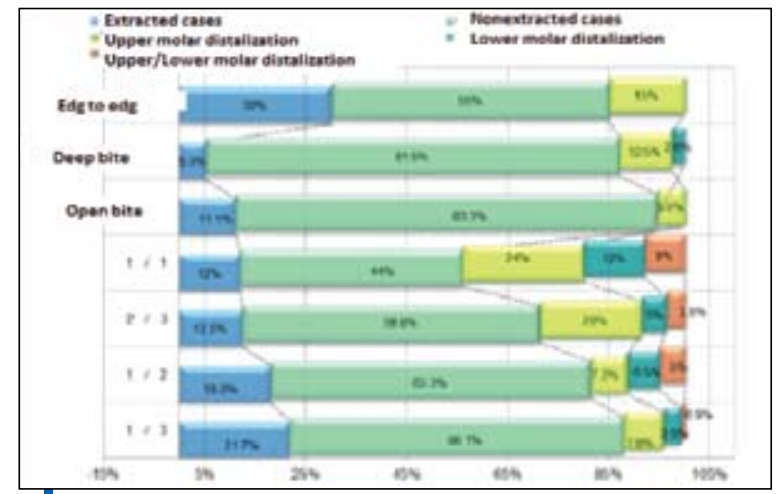

Figure 3. Correlation between

owerbite and distalization

Group B - represented by the rest of the patients, average age 12,15 were divided in two subgroups: B1 - cases of permanent teeth extractions and B2 - nonextraction cases, treated with other methods than distalization.

\section{Results}

The distribution of cases by gender demonstrated a predominance of female patients, representing $64 \%$ of the studied group (Figure 1).

Analysis of cases depending on the type of anomaly revealed a higher frequency of Angle Class I malocclusion (56,09\%), Class II represented by $35 \%$ of which $20,69 \%$ Class II/1, and 14,71\% Class II/2, and Angle Class III malocclusion represented only $8,51 \%$ of the studied group (Table 1).

Table 1. The distribution of anomalies

\begin{tabular}{|c|c|c|}
\hline Angle Class & No. cases & $\%$ \\
\hline Angle Class I anomalies & 244 & $56.09 \%$ \\
\hline Angle Class II/1 anomalies & 90 & $20.69 \%$ \\
\hline Angle Class II/2 anomalies & 64 & $14.71 \%$ \\
\hline Angle Class III anomalies & 37 & $8.51 \%$ \\
\hline Total & \multicolumn{2}{|c|}{435} \\
\hline
\end{tabular}

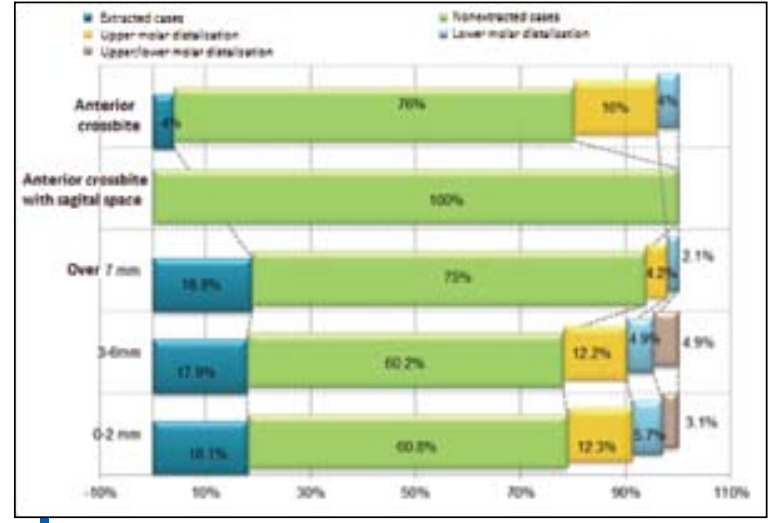

Figure 2. Correlation betwen owerjet and distalization

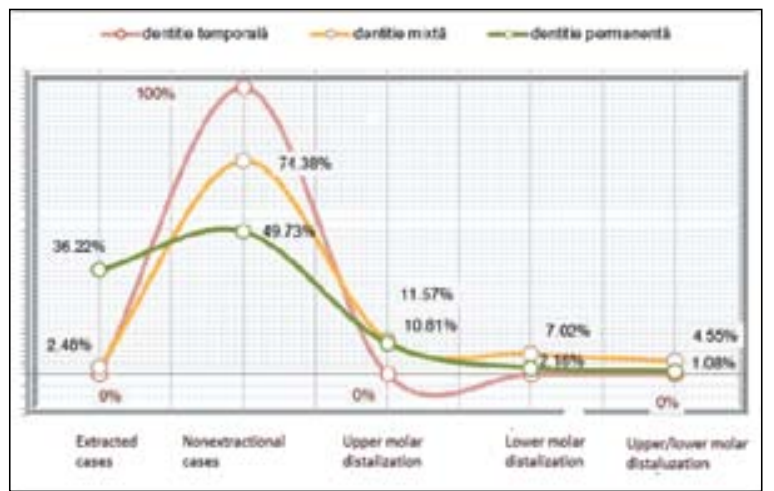

Figure 4. Correlation between molar

distalization and anomalies

In group A, represented by patients with molar distalization, the distribution on the arches was the following: the upper jaw $11,26 \%$, lower jaw $4,83 \%$ and bimaxilarry: $2.99 \%$ of cases (Table 2 ).

Table 2. The distribution of arches

\begin{tabular}{|c|c|c|}
\hline & No. cases & $\%$ \\
\hline Molar distalization & 83 & $19.08 \%$ \\
\hline Upper arch & 49 & $11.26 \%$ \\
\hline Lower arch & 21 & $4.83 \%$ \\
\hline Upper and lower & 13 & $2.99 \%$ \\
\hline Total & \multicolumn{1}{|c|}{166} \\
\hline
\end{tabular}

The distribution of cases from subgroup $B_{2}$ includes:

- upper expansion plate 34\%;

- lower expansion plate 8\%;

- maxillary disjunction (rapid palatal expander) 4\%;

- functional therapy 2,5\%;

- class II elastics 37\%;

- lee-way-space maintenance 1,5\%;

- stripping (interproximal reduction) 13\%.

Correlational analysis of the type of extractional/ nonextractional treatment related to overjet shows that: for overjet values between $0-2 \mathrm{~mm}$, 
the most frequent therapy is nonextractional (other than distalization) in $60 \%$ of cases, followed by dental extraction in $28 \%$ of cases and molar distalization in $12 \%$ of cases. The frequency with which distalization was used decreases with the growth of overjet value (Figure 2) .

Correlational analysis of the type of extractional/nonextractional treatment related to overbite shows that in open bite cases the extraction treatment is more frequent and in deep bite cases the most frequent treatment is nonextraction. (expander or stripping), followed by distalization cases (Figure 3 ).

Our study showed an increased incidence of therapy with molar distalization in Class II/2 anomalies $(28,13 \%)$, followed by Angle class I $(11,07 \%)$ and class II/1 (4,44\%) (Figure 4).

Regarding the type of dentition, we found that the difference in the incidence of upper molar distalization is not significant, between permanent $(10,81 \%)$ and mixed dentition $(11,57 \%)$, as opposed to the lower jaw, with a frequency of $7,02 \%$ in the mixed dentition and $2,16 \%$ in the permanent dentition (Figure 5 ).

A major issue in this kind of therapy is the timing of treatment initiation. In group $A$ the mean age of the patients was 11,25 years and in group B the mean age was 12,15 years.

The highest chances of molar distalization success are when the second molar has not yet erupted.

\section{Discussion}

The updated data from the literature indicates that during molar distalization we obtain a distal tipping and less corporal displacement because the force application point is at a distance from the resistance center of the tooth $(3,4)$. For bodily movement, the moment/force ratio at the molar centre of resistance must be zero, so it is necessary to reduce the moment on the molar bond using a counterbalancing couple (CBC) with effects in the vertical plane $(5,6)$.

\section{Bibliography}

1. Proffit WR. Biomechanics and mechanics. Contemporary Orthodontics 3rd ed. St Louis: Mosby Inc; 2000:298-305.

2. Baccetti T, Franchi L, Kim LH. Effect of timing on the outcomes of 1-phase nonextraction therapy of Class II malocclusion. Am J Orthod Dentofacial Orthop. 2009;136(4):501-509.

3. Antonarakis GS, Kiliaridis S. Maxillary molar distalization with noncompliance intramaxillary appliances in Class II malocclusion. A systematic review. Angle Orthod 2008, 78(6):1133-1140.

4. Escobar SA, Tellez PA, Moncada CA, Villegas CA, Latorre CM, Oberti G. Distalization of maxillany molars with the bone supported pendulum. $A$ clinical study.Am J Orthod Dentofacial Orthop 2007;131(4):545-549. 5. Henneman S, Von den Hoff JW, Maltha J.C. Mechanobiology of tooth movement. Eur J Orthod. 2008;30(3):299-306.

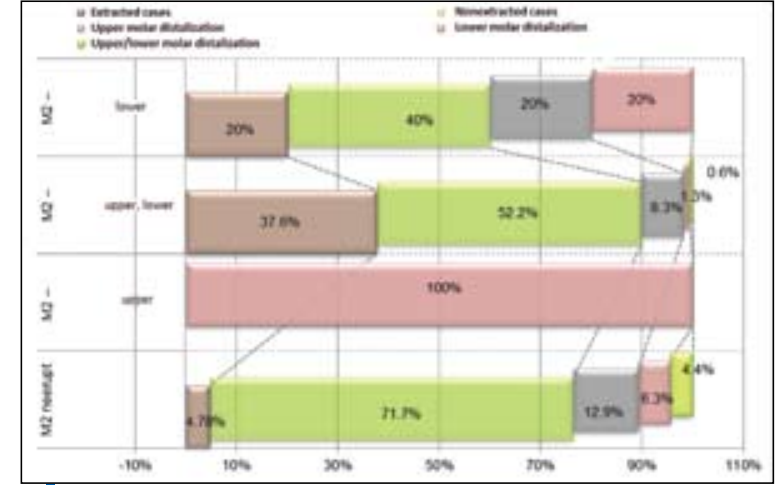

Figure 5. Correlation between type of dentition and molar distalization

In the orthodontic field it is better to have dental movement by translation (7).

But during distalization we obtain a distal tipping, is important to follow the maintenance of initial molar angulation, adding to the initial coronal tipping a root distal tipping (8).

Molar distalization is not a single orthodontic therapy, but has to be followed by fixed orthodontic treatment, which uses the obtained space for aligning the tooth and for overjet correction.

Most authors recommend that distalization appliances should be inserted on an oral part of the arch in order to be nearer to the resistance centre.

The other possibilities to have a translation movement during distalization is to put an extraoral force (9).

\section{Conclusions}

Molar distalization is a challenge in orthodontic treatment and is indicated for Angle II Class, crowding and low angle (extraction makes the profile worse).

The rate of success in molar distalization is less than that in other nonextraction methods and sometimes this method is followed by extraction.

6. Kinzinger GSM, Fritz UB, Sander FG, Diedrich PR. Efficiency of a pendulum appliance for molar distalization related to second and third molar eruption stage. Am J Orthod Dentofacial Orthop 2004; 125(1):8-23.

7. Birte M. Biological reaction of alveolar bone to orthodontic tooth movement. The Angle Ortodontist. 1999;69(2):151-158.

8.Papadopoulos MA,MavropoulosA, KaramouzosA.Cephalometric changes following simultaneous first and second maxillary molar distalization.J Orofac Orthop 2004;65 (2):123-136.

9. Klontz H. The Extraction/nonextraction dilemma - the Class II solution. The Tweed Profile. 2006;5:25-30.

10. KorkmazS, Fulya I, Ferdi A, Tülin A. Unilateral molar distalization with a modified slider. Eur J Orthod. 2006;28(4):361-365. 


\section{Mariana Păcurar}

Professor, DDS, PhD, University of Medicine and Pharmacy Târgu-Mureș, Faculty of Dentistry, Orthodontic Department, Târgu-Mureş, România

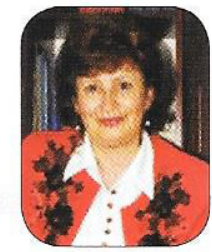

Mariana Pacurar is a full professor of Pedodontics and Orthodontics at the University of Medicine and Pharmacy Târgu-Mureş

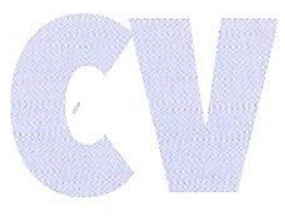
and dean of the Faculty of Dental Medicine.

In 1983 graduated from the Faculty of Dental Medicine (MD degree), the University of Medicine and Pharmacy Târgu-Mureş.

Doctor in Dental Medicine (PhD, 1999) at the University of Medicine and Pharmacy Târgu-Mureş. Competence in radiological diagnosis in dental medicine at University of Medicine and Pharmacy Cluj-Napoca (2000). She is head of Pediatric Dentistry Clinics at Târgu-Mureș, and also head of Pedodontics-Orthodontics Department of the Faculty of Dental Medicine in Târgu-Mureş.

She is author of more than 150 research papers and 7 books on several topics in orthodontics, pedodontics and general dentistry.

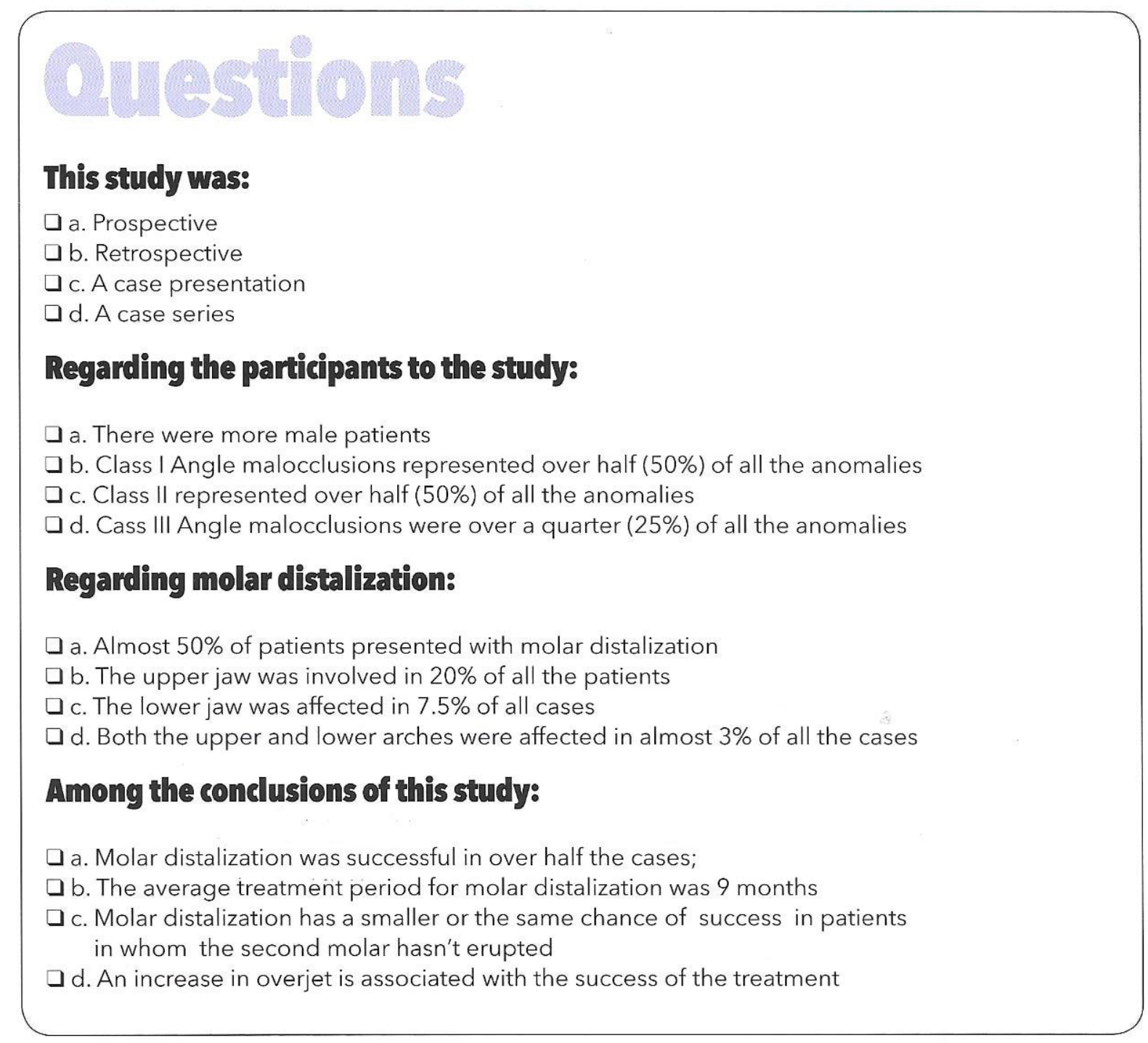

